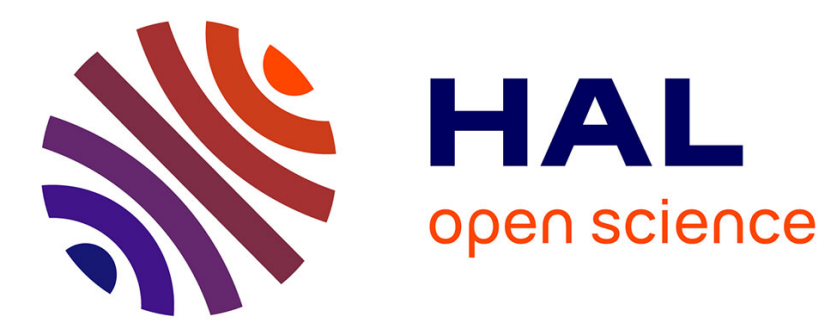

\title{
Influence of Ir Additions and Icosahedral Short Range Order (ISRO) on Nucleation and Growth Kinetics in Au-20.5Wt Pct Cu-4.5Wt PctAg Alloy
}

\author{
Julien Zollinger, B. Rouat, J. Guyon, S. K Pillai, M. Rappaz
}

\section{To cite this version:}

Julien Zollinger, B. Rouat, J. Guyon, S. K Pillai, M. Rappaz. Influence of Ir Additions and Icosahedral Short Range Order (ISRO) on Nucleation and Growth Kinetics in Au-20.5Wt Pct Cu-4.5Wt PctAg Alloy. Metallurgical and Materials Transactions A, 2019, 50, pp.2279-2288. 10.1007/s11661-01905176-2 . hal-02050947

\section{HAL Id: hal-02050947 https://hal.science/hal-02050947}

Submitted on 27 Feb 2019

HAL is a multi-disciplinary open access archive for the deposit and dissemination of scientific research documents, whether they are published or not. The documents may come from teaching and research institutions in France or abroad, or from public or private research centers.
L'archive ouverte pluridisciplinaire HAL, est destinée au dépôt et à la diffusion de documents scientifiques de niveau recherche, publiés ou non, émanant des établissements d'enseignement et de recherche français ou étrangers, des laboratoires publics ou privés. 


\title{
Influence of Ir additions and Icosahedral Short Range Order (ISRO) on nucleation and growth kinetics in $\mathrm{Au}-20.5 \mathrm{wt} \% \mathrm{Cu}-4.5 \mathrm{wt} \% \mathrm{Ag}$ alloy
}

\author{
J. Zollinger, B. Rouat, J. Guyon, S. K. Pillai, M. Rappaz \\ Accepted Manuscript in Metal. Mater. Trans. A
}

\begin{abstract}
Based on detailed EBSD analyses, Kurtuldu et al [1] have explained the grain refinement of Au-12.5 wt.\%Cu-12.5 wt.\%Ag (yellow gold) by the addition of minute amounts of Ir in terms of "icosahedral quasicrystal (iQC)-mediated nucleation", i.e., Ir induced the formation of Icosahedral Short Range Order (ISRO) of atoms in the liquid, leading to the formation of $\mathrm{QQC}$ on which the fcc-phase forms. In the present contribution, we show that: (i) this mechanism is also responsible of the grain refinement in $\mathrm{Au}-20.5$ wt. $\% \mathrm{Cu}-4.5$ wt. $\% \mathrm{Ag}$ (pink gold) with Ir addition; (ii) ISRO also influences the morphology and growth kinetics of the fcc phase: at solidification rate of a few $\mathrm{mm} / \mathrm{s}$, $\langle 100\rangle$ dendrites are replaced by a cellular-type morphology growing along $\langle 111\rangle$ when 100 wt.ppm of Ir is added to the melt; (iii) iQC-mediated nucleation is accompanied by a spinodal decomposition of the liquid, which is revealed at high cooling rate by the formation of $\mathrm{Cu}$-rich particles or dendrites, some of them being also twinned, in parallel to iQC-mediated grain refinement and twin formation.
\end{abstract}

\section{Introduction}

Kurtuldu et al. [1,2] have presented recently a new mechanism of nucleation in fcc alloys based on icosahedral quasicrystals (iQC) or clusters formation in the liquid phase and acting as pre-nuclei for the fcc phase. This so-called "iQC-mediated nucleation" mechanism was evidenced first in Al-20wt\% Zn:Cr [2], and then in Au-12.5wt\%Ag$12.5 \mathrm{wt} \% \mathrm{Cu}: \mathrm{Ir}$ [1]. It is still a conjecture but based on several strong experimental facts, mainly based on EBSD observations: (i) The addition of minute amounts of $\mathrm{Cr}$ in aluminum alloys or Ir in gold alloys drastically reduces the grain size with an abnormal fraction of twinned grain boundaries; (ii) Several assembly of nearest-neighbor $(\mathrm{nn})$ grains exhibit multiple-twins (MT) relationship, with a common $\langle 110\rangle$ direction; (iii) Up to $5 \mathrm{nn}$ fcc grains have MT relationship compatible with the symmetry of a decahedron and up to $9 \mathrm{nn}$ grains exhibit a MT symmetry compatible with an icosahedron, (iv) Some nn grains exhibit a MT relationship compatible with the facets of a 
so-called interlocked icosahedron. The formation of twins and MT relationships between nn grains become clear when heteroepitaxy between icosahedral and fcc phases are considered, with $\{111\}_{\text {fcc }}$-planes and $\langle 110\rangle_{\text {fcc }}$-directions perpendicular to 3 -fold and 2-fold symmetry axes of an icosahedron, respectively.

Approximant phases exhibiting icosahedral building blocks as well as iQC's are known in $\mathrm{Al}-\mathrm{Cr}$ and formation of $(\mathrm{Al}, \mathrm{Zn})_{7} \mathrm{Cr}$-type iQC are thermodynamically compatible in Al-Zn alloys even at low Cr composition [2, 3, 4]. Although Tsai-type QC have been identified in $\mathrm{Au}_{49} \mathrm{Al}_{34} \mathrm{Tm}_{17}$ [5], $\mathrm{Au}_{51} \mathrm{Al}_{34} \mathrm{Yb}_{15}$ and $\mathrm{Au}_{44} \mathrm{Ga}_{41} \mathrm{Yb}_{15}$ [6], and $\mathrm{Au}_{44.2} \mathrm{Ca}_{14.1} \mathrm{In}_{41.7}$ [7], there is no known intermediate phases between Ir and $\mathrm{Au}, \mathrm{Ag}$ or $\mathrm{Cu}$, and thus no 5-fold symmetry clusters or QC $[8,9,10]$. Yet, the potency of Ir- and $\mathrm{Ru}$-additions for the grain refinement of gold alloys is known in dental and jewellery industries for more than a century, mainly for yellow gold alloys [11, 12, 13], and this mechanism has been attributed recently to iQC-mediation [1]. A first question then arises: is the grain refinement with Ir additions to gold alloys sensitive to composition variations, such as $\mathrm{Al}-\mathrm{Zn}-\mathrm{Cr}$ alloys are? No literature has been found to determine whether such mechanism would be efficient for other $\mathrm{Au}-\mathrm{Cu}-\mathrm{Ag}$ gold alloys.

Minute additions decrease atoms mobility in the liquid phase by promoting Icosahedral Short Range Order (ISRO), as shown for $\mathrm{Cr}$ in Al-Zn alloys [14]. In liquid alloys having a known strong ISRO tendency such as intermetallic metallic glass formers, Hornfeck et al. reported 10-fold twinned dendrites nucleated on a decagonal QC in several systems ( $\mathrm{Ni}-\mathrm{Zr}, \mathrm{Cu}-\mathrm{Zr}$, Ni-Hf and ternary combinations of these four elements) [15]. The occurrence of such 10-fold twinning is highly sensitive to nucleation undercooling and associated growth rate. It is then reasonable to ask to what extent the nucleation mechanism proposed by Kurtuldu et al. is sensitive to nucleation undercooling and local solidification processing conditions?

In this paper, the $\mathrm{iQC}$-mediated nucleation mechanism is investigated in $\mathrm{Au}-20.5 \mathrm{Cu}-$ 4.5 Ag alloys (alloy compositions are given in wt.\% in this paper unless stated otherwise) when 100 wt.ppm Ir are added to the melt. At low cooling rate, the mechanism operates as reported previously, i.e., the grain size is reduced and the percentage of twin grain boundaries increases. However, in rapid solidification processing involving high nucleation undercooling and high solidification rate, the mechanism still operates for Au-rich grains while two unexpected effects were observed with $100 \mathrm{ppm}$ Ir additions: (i) the formation fo small $\mathrm{Cu}$-rich particles from the melt i.e., "divorced" nucleation of fcc $\mathrm{Au}$ and $\mathrm{fcc} \mathrm{Cu}$, (ii) a modification of the attachment kinetics resulting in a modification of the morphology and preferred growth directions from $\langle 100\rangle$ to $\langle 111\rangle$. The present results give a new insight into iQC-mediated nucleation and highlight the differences between Al-based and Au-based systems.

\section{Materials and methods}

Gold alloys with the composition $\mathrm{Au}-20.5 \mathrm{Cu}-4.5 \mathrm{Ag}$ (99.999\% purity, corresponding to Au-43.3 at.\% Cu-5.6 at.\%Ag), with and without 100 wt.ppm Ir additions, were supplied by PX Group. Two types of solidification experiments were performed. DSC solidification experiments with $100 \mathrm{mg}$ of each alloy, with and without Ir, were performed in a Setaram MHTC calorimeter at a rate $\dot{T}=-0.25 \mathrm{~K} / \mathrm{s}$. The Free Fall Droplet (FFD) 
rapid solidification experiment has been described and characterized previously [16]. In this case, $4 \mathrm{~g}$ of each alloy were melted by induction up to $1030^{\circ} \mathrm{C}\left(100^{\circ} \mathrm{C}\right.$ superheat). After the temperature was stabilized, the liquid droplet was allowed to fall on a copper substrate cooled by water (about $5 \mathrm{~cm}$ free fall). This experiment allows to reach substantial nucleation undercooling with typical values ranging from 15 to 25 $\mathrm{K}$ [17]. The associated cooling rate $\dot{T}$ is in the range $-10^{4} \mathrm{~K} / \mathrm{s}$ at the substrate/melt interface to $-10^{2} \mathrm{~K} / \mathrm{s}$ at the top of the solidified droplet.

After standard metallographic preparation, the samples were electrolytically polished with a LectroPol-5 apparatus from Struers and prepared by ion polishing with a Gatan PECS II apparatus ( $4 \mathrm{kV}$ for $12 \mathrm{~h}$ ). Samples were examined by SEM and OM for imaging, Energy Dispersive Spectroscopy (EDS) for chemical analysis. Electron BackScatter Diffraction (EBSD) for phase identification and orientation was performed on a FEI Quanta 600 F FEG-SEM equipped with a Brucker Si-drift Quantax EDS detector and a Nordlys high-speed EBSD camera for low resolution maps and on a Zeiss Auriga 40 FEG-SEM equipped with an Oxford Instrument Symmetry camera for high resolution maps. The accuracy to identify twin grain boundaries in EBSD reconstructed microstructures was set to $5 \mathrm{deg}$. as in [1,2]. For the samples melted and solidified in the DSC calorimeter, the whole surface of the samples was analyzed by EBSD. For the samples processed with FFD, this analysis was performed over a surface 4 times larger than that shown in the Results section $\left(\approx 16 \mathrm{~mm}^{2}\right)$.

As will be shown in the next section, Cu-rich particles observed in the alloy with Ir have a characteristic size that is substantially lower than the size of Au-rich grains analyzed by EBSD. In order to have statistically significant microstructural information, an area of $4 \mathrm{~mm}^{2}$ was characterized using the methodology of Jeanmaire et al. [18]. Using a 2000 magnification required to detect the smaller $\mathrm{Cu}$-rich particles, the analyzed surface corresponds to about 2000 BSE-SEM images. With a software specifically developed for particle analysis, the sample stage can be driven and the sample completely analyzed automatically. For each detected $\mathrm{Cu}$-rich particle, the spatial coordinates, mean equivalent diameter and surface fraction are determined by image analysis.

Thermodynamic calculations were performed with the ThermoCalc software [19]. The thermodynamic parameters for the $\mathrm{Au}-\mathrm{Cu}, \mathrm{Ag}-\mathrm{Au}, \mathrm{Ag}-\mathrm{Cu}$ and $\mathrm{Ag}-\mathrm{Au}-\mathrm{Cu}$ systems were taken from Sundmann et al., Hassam et al. and Kusoffsky, respectively [20, 21, 22], and from Dinsdale for pure elements [23].

\section{Results}

\section{1 iQC-mediated nucleation in Au-20.5Cu-4.5Ag at low $\dot{T}$}

Without Ir addition, the specimen solidified at low cooling rate in the DSC apparatus exhibits very large grains, typically about $2 \mathrm{~mm}$ in size (i.e., one or two grains present in the analyzed surface). With $100 \mathrm{wt} . \mathrm{ppm} \mathrm{Ir}$, the grain size is drastically reduced to about $125 \mu \mathrm{m}$ as shown in Fig. 1 (see also Table 1). Together with this grain size reduction, about $7.6 \%$ of grains boundaries correspond to twins, as shown in Fig. 1, a value about 60 times larger than the $0.13 \%$ of twins in a random population of grains (with 5 deg. accuracy detection) [24]. This shows that Ir has a very similar effect in 
$\mathrm{Au}-20.5 \mathrm{Cu}-4.5 \mathrm{Ag}$ (pink gold), which contains more $\mathrm{Cu}$ and less $\mathrm{Ag}$ as compared with $\mathrm{Au}-12.5 \mathrm{Cu}-12.5 \mathrm{Ag}$ (yellow gold) [1]. It has to be mentioned that the cooling rate in [1] was unfortunately not specified, but considering the casting conditions in a copper mold, it is substantially higher than that of DSC (10 to 100 times higher).

\section{2 iQC-mediated nucleation in Au-20.5Cu-4.5Ag at high $\dot{T}$}

Figure 2 shows the microstructure of $\mathrm{Au}-20.5 \mathrm{Cu}-4.5 \mathrm{Ag}$ with and without Ir additions solidified at high cooling rate using the FFD experiment and observed using BSE-SEM. In all the figures related to FFD results, the thermal gradient goes from left to right, i.e.,
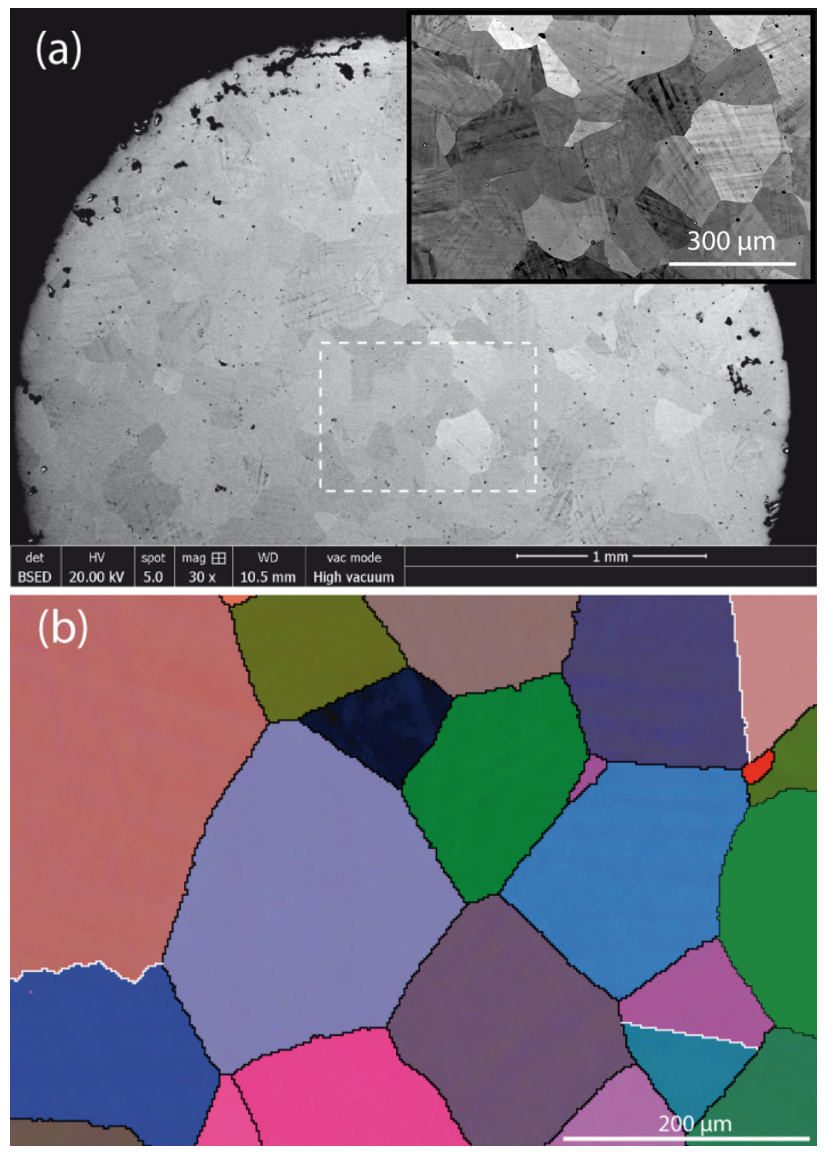

Figure 1: (a) Overall and enlarged view and (b) EBSD false colors map showing the microstructure of Au-20.5Cu-4.5Ag:100 wt.ppm Ir solidified in DSC. The view analyzed by EBSD is shown with a white rectangle and the EBSD colors of the grains correspond to their three measured Euler angles. Twin grain boundaries are indicated by white lines. 

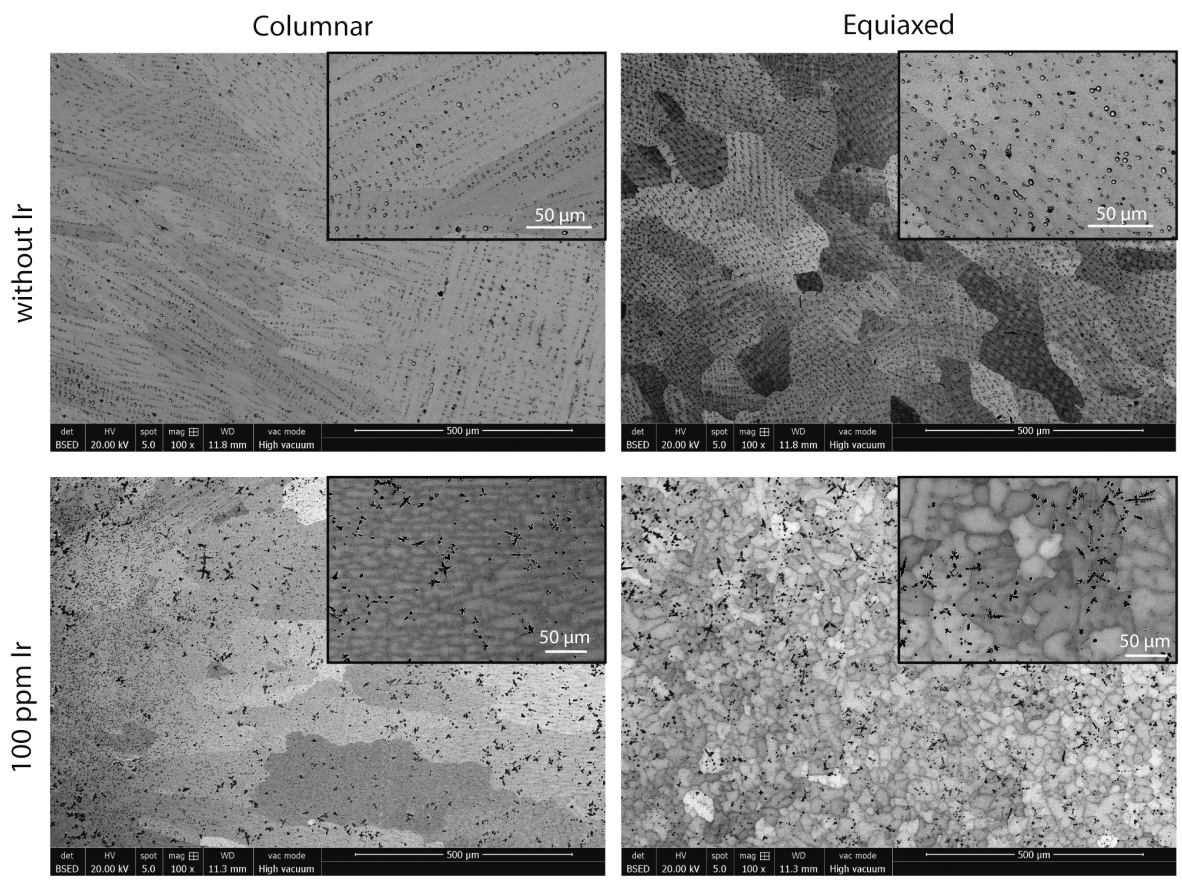

Figure 2: Backscattered electrons image showing the microstructure of $\mathrm{Au}-20.5 \mathrm{Cu}$ - 4.5Ag specimens rapidly solidified without (top) and with (bottom) 100 wt.ppm Ir. The left and right figures correspond to the columnar and equiaxed zones, respectively. For each figure, an enlarged view is shown in the small inserts.

the copper substrate is on the left. For both compositions, two regions of the droplets are shown: the region in contact with the copper substrate exhibits columnar grains, while near the top free surface where the cooling rate is estimated to be around -100 $\mathrm{K} / \mathrm{s}$ [16], the structure is equiaxed. For each micrograph, an enlarged view is shown in a small insert.

The Ir-free alloy solidified at high cooling rate clearly exhibits a dendritic fcc Aurich phase in both the columnar and equiaxed regions. As we shall see in the next section, microsegregation in gold alloys is not very strong but nevertheless, the liquid becomes richer in $\mathrm{Cu}$ and $\mathrm{Ag}$ as the solid fraction increases. As a result, the interdendritic regions for both the columnar and equiaxed zones are $\mathrm{Cu}$ - and $\mathrm{Ag}$-rich and appear darker in BSE. Looking at the small inserts, the typical dendrite arm spacing of this specimen is very fine, typical of a high cooling rate (about 5 and $10 \mu$ in the columnar, respectively equiaxed zone of Fig. 2).

Under the same conditions, the addition of 100 wt.ppm Ir modifies the microstructure in several ways. First of all, the columnar zone is more extended than in the Ir-free specimen, the grains look less elongated and have an internal microstructure which is no longer dendritic. We will come back to this point in the next section. The equiaxed region exhibits much finer grains, thus confirming the influence of Ir on nucleation even 
at high cooling rate. Since the grains are much smaller (27 $\mu \mathrm{m}$, Table 1) as compared with the Ir-free specimen $(99 \mu \mathrm{m})$, they have a globular-dendritic, rather than dendritic morphology. The characteristic length scale of both the columnar and equiaxed microstructures is much larger than the dendrite arm spacing of the Ir-free specimen. For the equiaxed grains, it is comparable with the grain size itself, i.e., the grains are nearly globular.

Besides its influence on the size and morphology of the Au-rich phase grains, the addition of 100 wt.ppm of Ir in $\mathrm{Au}-20.5 \mathrm{Cu}-4.5 \mathrm{Ag}$ induces at high cooling rate the formation of very fine particles, sometimes dendrites. They appear black in BSE contrast and their composition determined by EDS corresponds to more than 95 at. $\% \mathrm{Cu}$. They are not located at interdendritic/intergranular regions of columnar/equiaxed grains and are definitely too coarse to be associated with a solid-state transformation. Their formation is further discussed in the next section.

Figure 3 shows the results of the EBSD analysis in the columnar-to-equiaxed transition (CET) for the alloy without (a) and with (b) $100 \mathrm{wt} . p p m$ Ir. Surprisingly, the extension of the columnar zone of the Ir-free alloy $(500 \mu \mathrm{m})$ is smaller than that of the alloy with Ir $(1100 \mu \mathrm{m})$, while the average grain size of the equiaxed grains without Ir $(99 \mu \mathrm{m})$ is about four times larger than that with Ir $(27.4 \mu \mathrm{m}$, see Table 1). For both specimens, the evolution of the average grain size as a function of the distance from the copper substrate is shown in Figure 3(c). Please note that in the columnar zone, the size is an average between the extension of the grains along the thermal gradient and their transverse direction. In the Ir-free specimen, the grain size does not evolve much over the whole thickness of the droplets. In the alloy with Ir, the grain size first increases in the columnar zone as a result of grain competition. It then decreases drastically at the CET down to below $30 \mu \mathrm{m}$ and remains about constant in the whole equiaxed zone.

In the Ir-free specimen, there are very few twinned grain boundaries (only one is visible in the columnar zone of Figure 3(a)). The percentage of these twins in the equiaxed zone is about $0.13 \%$ (see Table 1), i.e., a value in close agreement with that of a random distribution of fcc grains [24] despite the small number of grains analyzed $(\approx 500$ grains). For the specimen with Ir (Figure 3(b)), the percentage of twinned grain boundaries in the equiaxed zone is much larger and close to the value found in the DSC specimen (see Table 1). There are very few twinned grain boundaries in the columnar zone as for the Ir-fee specimen. In the equiaxed zone, the fraction of twins evolves as a function of the distance from the copper substrate (Figure 3(c)): it first increases to reach a maximum at about $2.7 \mathrm{~mm}$ from the copper substrate, before decreasing near the top of the drop. This tends to indicate a sensitivity to the cooling rate of the iQC-mediated nucleation mechanism, although the average value is close to the value found for the same alloy solidified in DSC. Please note that the value reported in Table 1 corresponds to the fraction of twins in the equiaxed zone only, but for the entire analyzed surface (i.e., 4 times the surface shown in Figure 3).

In order to prove that the high frequency of twinned grain boundaries observed in $\mathrm{Au}-20.5 \mathrm{Cu}-4.5 \mathrm{Ag}$ with 100 wt.ppm Ir is associated with an iQC-mediated nucleation mechanism similar to what has been observed in $\mathrm{Al}-\mathrm{Zn}: \mathrm{Cr}$ [2] or $\mathrm{Au}-12.5 \mathrm{Cu}-12.5 \mathrm{Ag}$ (yellow gold) [1], it is necessary to find sets of nn grains having multiple twin or neartwin (MT) relationships which reproduce the symmetry of the icosahedron. With a 5 deg. tolerance, many sets of 3 or 4 such nn grains can be found in the equiaxed zone of 
(a)
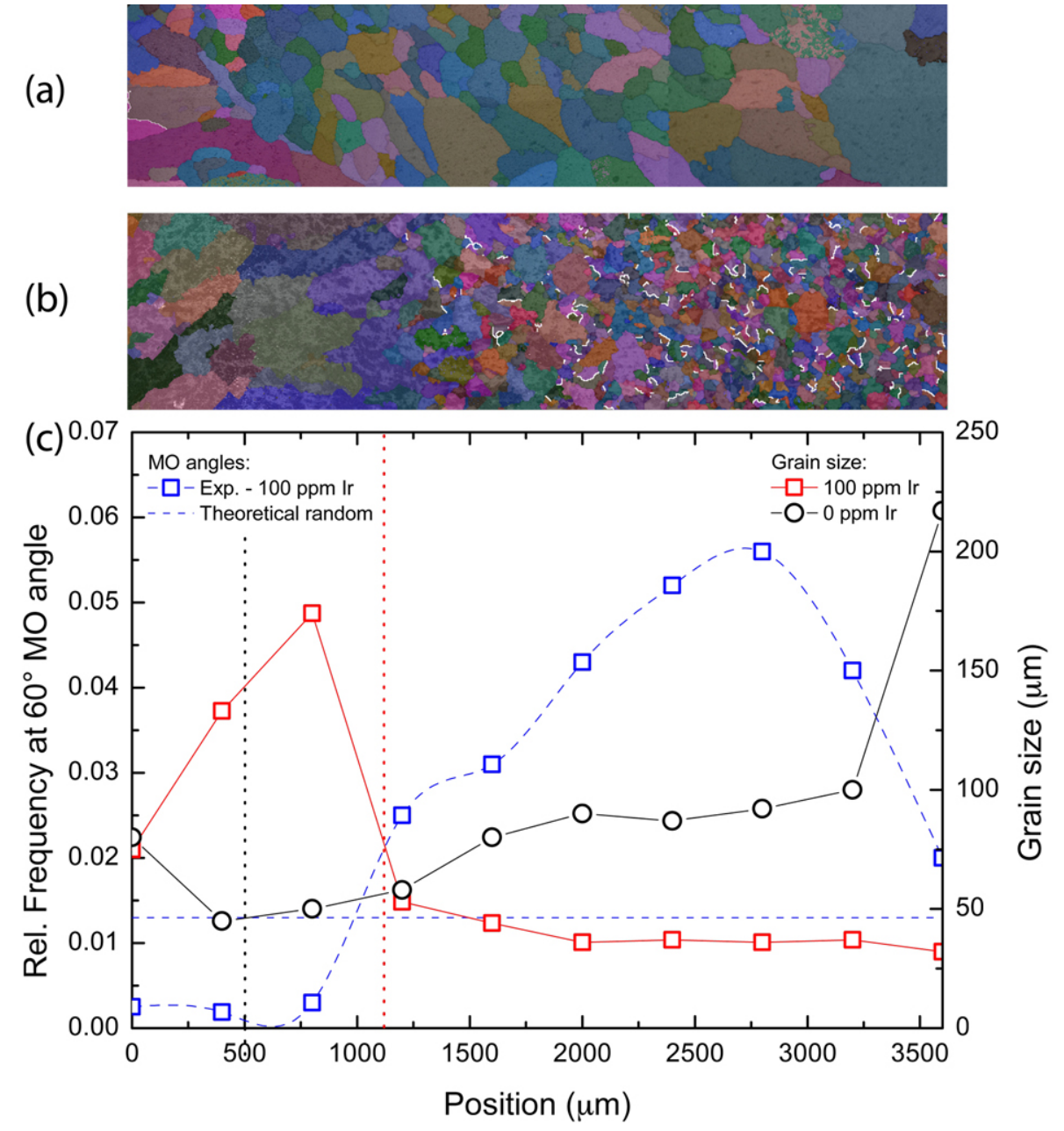

Figure 3: False colors EBSD map of $\mathrm{Au}-20.5 \mathrm{Cu}-4.5 \mathrm{Ag}$ rapidly solidified (a) without Ir and (b) with 100 wt.ppm iridium. In (c), the grain sizes deduced from (a) and (b) are plotted as a function of the distance from the copper substrate. The fraction of twinned grain boundaries for the specimen with Ir is also reported in this figure. The vertical dashed lines correspond to the position of the CET with (red) and without (black) Ir additions. The horizontal black line corresponds to the Mackenzie value of twins in a randomly oriented population of grains, with accuracy of $5 \mathrm{deg}$. for their detection.

the FFD specimen. To detect more nn grains with such MT relationships, the tolerance has to be increased in order to account for subgrain misorientations induced by the $7.35 \mathrm{deg}$. aperture default angle associated with the decahedral configuration of $5 \mathrm{fcc}$ tetrahedra.

Setting the tolerance to 7.5 deg., Figure 4 (a) shows a set of $9 \mathrm{nn}$ grains which re- 

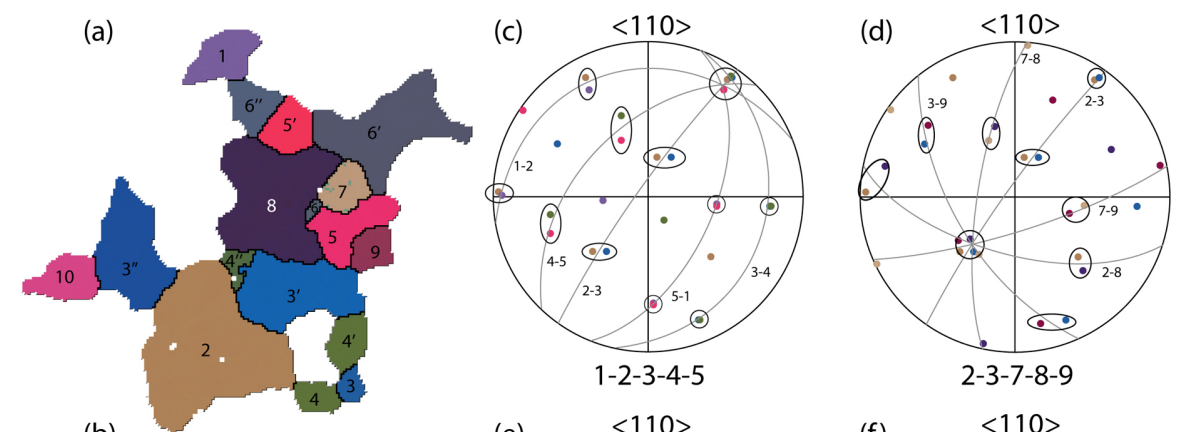

(b)
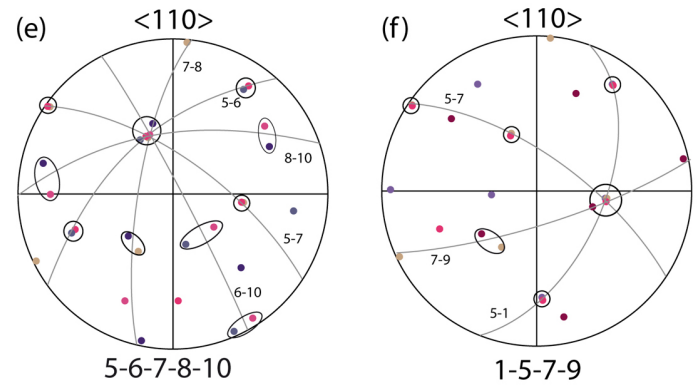

Figure 4: (a) False colors EBSD map of $9 \mathrm{nn}$ grains within the equiaxed zone of rapidly solidified Au-20.5Cu-4.5Ag with 100 wt.ppm Ir, showing multiple twin or near-twin (MT) relationships compatible with the icosahedron symmetry. Each $\langle 110\rangle$ pole figure shown in (c)-(f) corresponds to a set of 5 (4 in (f)) nn grains having a common $\langle 110\rangle$ axis and MT relationships, i.e., assumed to form on the 5 (4 for (f)) triangular facets of the icosahedron surrounding a 5-fold symmetry axis. Common $\{111\}$ twin or near-twin planes are shown with arcs of circle, while common $\langle 110\rangle$ directions are surrounded with small circles or ellipses. In (b), a comparison is made between the orientation of the common $\langle 110\rangle_{c-f}$ directions of the 4 sets of grains whose pole figures are shown in (c)-(f) (blue squares) with the 5-fold symmetry axes of the icosahedron template having the closest orientation (red pentagons).

produce the symmetry of an icosahedron when fcc tetrahedra are set on its triangular facets with the heteroepitaxy relationship found in [2], i.e., $\langle 111\rangle_{\text {fcc }} / /\langle 3$-fold symmetry axis $\rangle_{\text {ico }}$ and $\langle 110\rangle_{\text {fcc }} \perp\langle 2 \text {-fold symmetry axis }\rangle_{\text {ico. }}$. The grains identified by EBSD are displayed with false colors which are then used in the four $\langle 110\rangle$ pole figures of Fig. 4(c)-(f). In (c), the grains 1, 2, 3, 4 and 5 have MT relationships outlined by the five $\{111\}$ common planes (arcs of circle) with $\langle 110\rangle$ common directions (surrounded by small circles or ellipses for near-twins). The five $\{111\}$ twin planes have a common $\langle 110\rangle$ direction which we label $\langle 110\rangle_{c}$. The same occurs for grains 2, 3, 7, 8 and 9 in Fig. 4(d) with a common $\langle 110\rangle_{d}$ direction, for grains 5, 6, 7, 8 and 10 in Fig. 4(e) with a common $\langle 110\rangle_{e}$ direction, and finally for grains 1, 5, 7 and 9 (only 4 grains) in Fig. 4(f) with a common $\langle 110\rangle_{f}$ direction.

This clearly shows that each of the four sets of 5 (or 4 ) nn grains whose pole figures are shown in (c)-(f) are in MT relationship with a common $\langle 110\rangle$ direction correspond- 
ing to the 5-fold symmetry axis of a decahedron (assembly of 5 tetrahedra with 10 triangular facets). Moreover, these four sets of grains correspond to a single icosahedron template. If such is the case, the angles between the common $\langle 110\rangle_{c-f}$ directions should correspond to those between the 5-fold symmetry axes of the icosahedron. In an icosahedron, these angles are either $63.43 \mathrm{deg}$. (first vertex neighbors) or 116.57 deg. (second vertex neighbors). The mutual angles found between the four common $\langle 110\rangle_{c-f}$ directions of Fig. 4, as identified by the crossing of the $\{111\}$ planes, are: $60.3,66.2,72.9,78.2,79.2$ and 125.7 deg.. The comparison between the common $\langle 110\rangle_{c-f}$ directions of the sets of fcc grains and the 5-fold directions of the assumed icosahedron template is shown in Fig. 4(b). Considering the cumulated aperture default angle of fcc grains around a common 5-fold symmetry axis of an icosahedron (7.35 deg.), subgrain misorientations induced during growth and measurement uncertainty, this comparison is a fairly strong evidence that ISRO is again responsible of the high twinning frequency of grain boundaries in this specimen.

\subsection{Nucleation, grain distribution and stability of fcc-Cu particles}

Figure 5 shows a BSE-SEM image corresponding to Figure 3(b), i.e., Au-20.5Cu$4.5 \mathrm{Ag}$ with $\mathrm{Ir}$ solidified rapidly, and the corresponding density of $\mathrm{Cu}$-rich particles (or dendrites when they are bigger) as a function of the distance from the copper substrate. The density of particles was measured over the surface using $0.2 \mathrm{~mm}$ steps. Again the vertical red dotted line corresponds to the position of the CET of the fcc-Au grain structure. The evolution of the number of $\mathrm{Cu}$-rich particles decreases with increasing distance from the substrate, and thus decreasing cooling rate. At the top of the sample where the cooling rate is "only" about $-100 \mathrm{~K} / \mathrm{s}, 740$ particles $/ \mathrm{mm}^{2}$ were observed.

In order to check the $\mathrm{Cu}$-rich phase stability, different heat treatment were performed between 700 and $900^{\circ} \mathrm{C}$ with $50^{\circ} \mathrm{C}$ steps for $1 \mathrm{~h}$ with a dynamic argon atmosphere and furnace cooled. At 700 and $750^{\circ} \mathrm{C}$, the microstructure does not evolve significantly and only a smoothing of the dendrites envelope was observed. Between 800 and $850^{\circ} \mathrm{C}$, the fraction of $\mathrm{Cu}$ particles start to decrease. It appears that they have partially melted with an increasing fraction of liquid, as could be seen from the pores formed near these remelted particles. Although the temperature is much lower than the melting point of pure copper, diffusion lengths on the order of $1 \mu \mathrm{m}$ were measured. Therefore, local diffusion tends to equilibrate the compositions and thermodynamic calculations indicate that partial melting should occur in this alloy. At $850^{\circ} \mathrm{C}$, about $50 \%$ of the particles have disappeared by this local diffusion-remelting process. At $900^{\circ} \mathrm{C}$, part of the sample has melted and there is no $\mathrm{Cu}$-rich-phase remaining after cooling.

\section{Discussion}

\section{1 iQC-mediated nucleation of the Au-phase}

It is recalled that the iQC-mediated nucleation mechanism of fcc metals proposed by Kurtuldu et al. [2] considers that 20 new fcc grains can form on the facets of an icosa- 


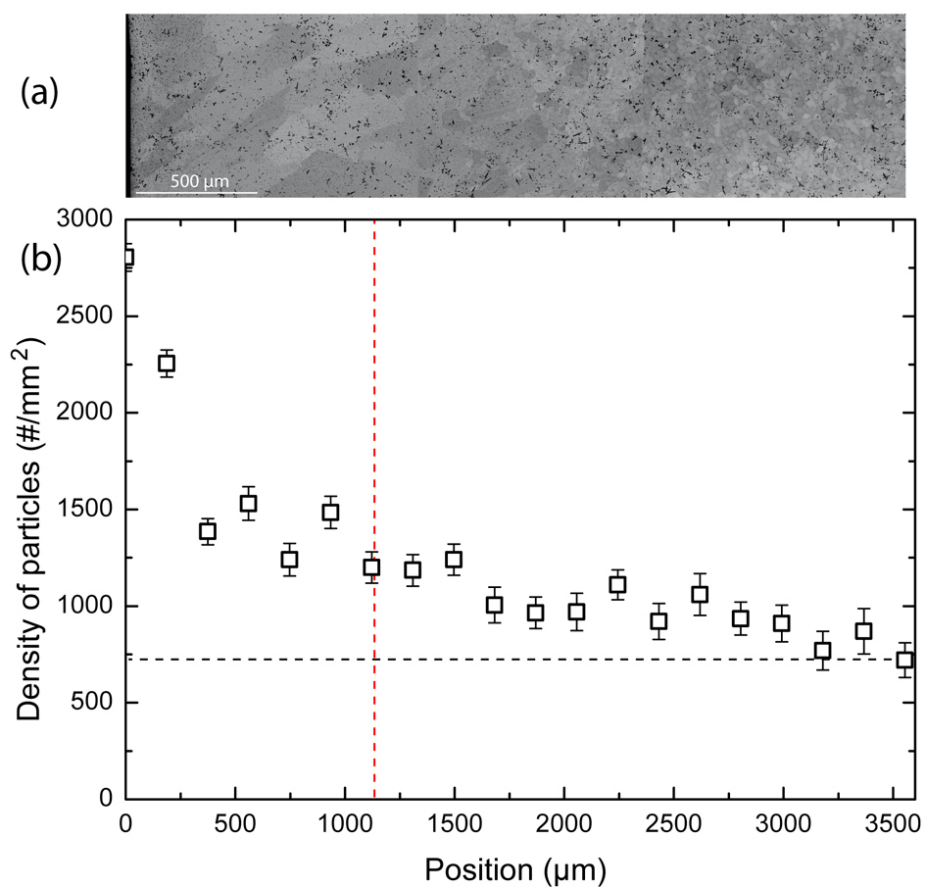

Figure 5: (a) BSE-SEM image corresponding to Figure 2(b), i.e., $\mathrm{Au}-20.5 \mathrm{Cu}-4.5 \mathrm{Ag}$ rapidly solidified with 100 wt.ppm Ir, and (b) Surface density of Cu-rich particles/dendrites detected by image analysis. The red dotted line indicates the position of the CET in this specimen. The horizontal dashed black line corresponds to the minimum density of particles measured at $3500 \mu \mathrm{m}$ from the copper substrate.

hedron with heteroepitaxy relationships where $\{111\}_{\text {fcc }}$ planes are parallel to the facets of the icosahedron and the $\langle 110\rangle_{\text {fcc }}$ directions are parallel to their edges. This leads to $20 \mathrm{fcc}$ grains which have multiple twin relationships, but with a default angle of 1.5 degree at each twin plane. During growth, these grains tend to re-arrange so as to minimize interfacial energy: small rotations, frequently observed as subgrain misorientations during solidification, tend to accumulate these default angles into one or two single boundaries. This leads to "perfect" twinning and "near-twinning" relationships between the grains, where the last orientation relationship corresponds to a twin and a rotation of 7.5 degree around a common $\langle 110\rangle_{\text {fcc }}$ direction.

From the results presented before, we can conclude first that the iQC-mediated nucleation induced by Ir additions, revealed first in $\mathrm{Au}-12.5 \mathrm{Cu}-12.5 \mathrm{Ag}$ (yellow gold) [1], is also effective in the equiaxed zone of $\mathrm{Au}-20.5 \mathrm{Cu}-4.5 \mathrm{Ag}$ (pink gold). The fraction of twinned grain boundaries (around 7.5\% in both the slowly- and rapidly-cooled specimens) is a first indication. For comparison, Kurtuldu et al. [1] found about $11 \%$ of twinned grain boundaries in $\mathrm{Au}-12.5 \mathrm{Cu}-12.5 \mathrm{Ag}$ with $100 \mathrm{wt} . \mathrm{ppm} \mathrm{Ir}$ and a grain size comparable to that of $\mathrm{Au}-20.5 \mathrm{Cu}-4.5 \mathrm{Ag}$ solidified rapidly. The cooling rate is un- 
fortunately not mentioned in [1], but is probably close to that of the FFD specimen since $\mathrm{Au}-12.5 \mathrm{Cu}-12.5 \mathrm{Ag}$ was cast in a copper mold. A stronger indication to validate the conjecture of $\mathrm{iQC}$-mediated nucleation is that many nearest-neighbor grains are in a configuration of multiple twinning compatible with the icosahedral symmetry, as shown for example in Fig. 4.

In the columnar zone, there are very few twins, regardless whether Ir is added or not. It does not mean that the iQC-mediated mechanism did not operate at the onset of columnar growth near the copper chill in the specimen with Ir. But as growth proceeds, grain competition selects those having a $\langle h k \ell\rangle$ crystallographic direction most closely aligned with the thermal gradient, $\langle k h \ell\rangle$ being normally $\langle 100\rangle$ for cubic metals. Because of the angles between the 3-fold facets of an icosahedron, only one of the potentially $20 \mathrm{fcc}$ grains forming on an iQC can have such an orientation. Therefore, it can be assumed reasonably that columnar grains selected after some growth distance originate from separate iQC's and should not exhibit any twin, except those than can be detected within the preset accuracy among randomly oriented grains. (As a matter of fact, a perfect $\langle 100\rangle$ texture cannot exhibit any twin due to the cubic symmetry).

Several questions nevertheless remain. Why does the fraction of twinned grain boundaries vary as a function of the distance from the copper chill (or cooling rate) in the FFD experiment (between 2 and 6\% in Fig. 3(c)), while the average fraction measured over a larger surface is close to that of the DSC specimen (see Table 1)? And why this fraction of twins varies non-monotonously while the size of equiaxed grains remain fairly constant, with a slow gradual decrease from about $50 \mu \mathrm{m}$ to about $35 \mu \mathrm{m}$ ? Kurtuldu et al. [1] have shown that the grain size correlates better with the fraction of twinned grain boundaries than with the amount of Ir added to the melt. Such seems not to be the case here. Finally, why is the columnar zone more extended and the corresponding grain size larger when Ir is added to the melt, while the equiaxed zone is drastically grain refined? It is well-known in Al alloys that the addition of $\mathrm{TiB}_{2}$ inoculant particles refines the grain size and at the same time reduces, or even eliminates the columnar zone, a mechanism very well explained by simple criteria such as derived by Hunt [25].

The answers are not simple since the final grain structure in solidification is a convolution between nucleation and growth kinetics acting concomitantly in a non-steady thermal field. Without Ir, these mechanisms are well understood because both the columnar and equiaxed grains clearly exhibit a dendritic structure (see Fig. 2(top)), for which the growth kinetics is fairly well known and about the same for both morphologies. Knowing the growth kinetics, so-called micro-macroscopic approaches allow to interpret the results and give access to the nucleation distribution [26]. With Ir (Fig. 2(bottom)), not only the grain size in the equiaxed zone is drastically reduced, but the microstructure of both columnar and equiaxed grains is clearly no longer dendritic. It is in fact much coarser as compared with the dendrite arm spacing of the Ir-free specimen. In the equiaxed zone, the grains are globular-dendritic and this could be due to the small grain size. (It is to be noted that there is no growth kinetic model for such morphology besides the phase field method). For columnar grains, the microstructure is astonishing: it looks like small cells growing more or less in the direction of the thermal gradient.

It could be thought that the formation of $\mathrm{Cu}$ particles, which occurs first, is respon- 

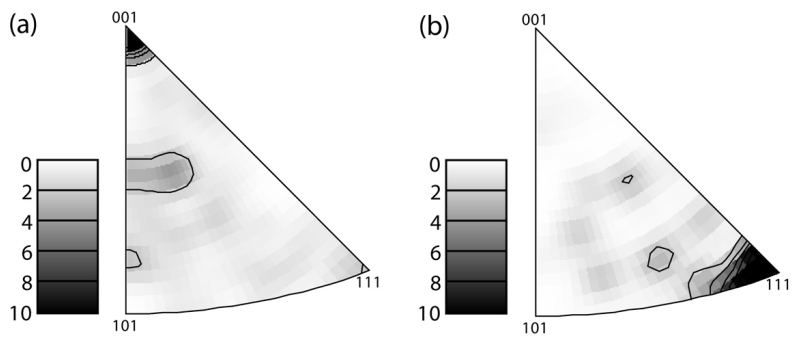

Figure 6: Inverse texture pole figures of the columnar zone of the FFD specimens without (a) and with $100 \mathrm{ppm}$ Ir (b), shown in the unit triangle $\langle 001\rangle-\langle 101\rangle-\langle 111\rangle$ of the fcc structure. In these stereographic projections, the grey level indicates the probability of having the thermal gradient direction aligned along a certain crystallographic direction of the Au-rich fcc grains. The texture density is scaled between 0 and 10 for both specimens.

sible for this change of morphology of the Au-rich columnar grains when Ir is added. Although these particles represent about $4 \%$ in volume, this does not influence much the composition of the liquid, and thus microsegregation when the Au-rich phase forms (i.e., about $2 \mathrm{wt} \%$ less $\mathrm{Cu}$ in the liquid). On the other hand, their density being fairly high (Fig. 5), they could possibly influence (or "drive") the growth direction of the Au-rich phase, as it has been shown for example in [27]. However, Figure 6 clearly rules out this mechanism and shows that this change of morphology, and thus of growth kinetics, of columnar grains is directly induced by Ir addition.

Figure 6 shows the corresponding textures of the columnar zone of the FFD Au20.5 Cu-4.5Ag specimens, without (a) and with $\operatorname{Ir}(\mathrm{b})$. In these Inverse Pole Figure (IPF) texture representation, the thermal gradient direction, i.e., normal to the copper chill substrate, is represented with respect to the orientation of the grains composing the columnar zone. Considering the fcc symmetry, IPF are represented in a stereographic projection limited to the unit triangle $\langle 001\rangle-\langle 101\rangle-\langle 111\rangle$. The dendritic morphology of the Ir-free specimen has a very strong $\langle 100\rangle$ texture, i.e., the columnar grains have one of their $\langle 100\rangle$ directions aligned with the thermal gradient direction, as revealed by the high intensity (black) near $\langle 001\rangle$ in Fig. 6(a). This is expected from the grain competition occurring within the columnar zone of "normal" fcc alloys, where $\langle 001\rangle$ dendrites well aligned with the thermal gradient have the lowest velocity, and thus the smallest undercooling. With Ir (Fig. 2), the peculiar microstructure of the columnar grains exhibits a $\langle 111\rangle$ texture (Fig. 6(b)). This was totally unexpected and clearly confirms that ISRO in the liquid not only contributes to nucleation via the $\mathrm{iQC}$-mediated mechanism, but also modifies the growth morphology and texture via an attachment kinetics contribution. If one considers that icosahedral clusters in the liquid have to orient themselves in order to attach 3-fold symmetry facets, i.e., 3 atoms, to the growing fcc solid, this would explain why this favors the growth of (111) planes parallel to the isotherms.

This "ISRO-influence" on the growth kinetics and morphology of the fcc phase is most probably responsible of peculiar morphologies in Al-alloys previously reported. 
For example, Henry et al [28] have observed the formation of "curly" $\langle 112\rangle$ dendrites or the formation of fractal-type secondary arms in commercial $\mathrm{Al}-\mathrm{Cu}-\mathrm{Mg}$ alloys. Kurtuldu [29] has seen that the growth directions of trunks and arms in Al-Zn:Cr change with the solidification speed. Bedel et al [30] have observed the formation of $\langle 111\rangle$ dendrites in small Al-Cu droplets rapidly solidified. At the light of our present results, all these observations start to make sense if one considers the attachment kinetics of icosahedral clusters, rather than individual atoms, at the interface of the fcc phase with the liquid.

A similar ISRO-influence on growth could also explain the peculiar arrangement of some of the MT equiaxed grains observed in Fig. 4(a). Some of them appear at several locations with an alternated sequence, e.g., grains 3 and 4 appear in a sequence $4-3-4^{\prime}-3$ ' -4 ''-3"' around grain 2, or grains 5 and 6 in the sequence 6-5-6'-5'-6" around grains 7 and 8. This was not the case in Figure 6 of [1]. We could first think that the complex 3D internal microstructure of the grains is such that grains appear as several patches in metallographic cross sections, as can be the case for well-developed fully dendritic specimens. But this looks quite improbable, especially with the fairly compact globular-cellular morphology observed in Fig. 2. A more plausible explanation could be again the influence of ISRO on growth, and not (or not only) on nucleation, as suggested by preliminary atomistic calculations [31]. If an iQC template can indeed lead to the formation of 20 MT grains, as suggested by Kurtuldu et al. [1, 2], the reverse heteroepitaxial relationship could also act during growth.

Considering that ISRO "motives" or "bricks" in the liquid phase can attach to a single growing fcc grain with the same heteroepitaxy relationship, this situation becomes similar to a local stacking fault during growth: the next (111) layer forming on the stacking fault transforms this defect into a twin. In the case of an ISRO motive attaching to the fcc grain, another (111) layer can form on another facet of the icosahedron motive leading to the formation of a twin but with a misorientation of 1.5 degree. In the present observations, this process can explain the alternance of twinned grains observed in the EBSD pictures. As far as the texture and morphology of columnar grains are concerned, it is assumed that the $\langle 111\rangle$ texture and very peculiar morphology observed at high solidification rate with Ir addition is also due to this attachment of ISRO motives for two reasons: (i) the presence of ISRO decreases the mobility of atoms in the liquid phase, as measured by Kurtuldu et al. for $\mathrm{Al}-\mathrm{Zn}: \mathrm{Cr}$ [14], thus introducing an attachment kinetics contribution revealed at much lower solidification speed than for "ISRO-free" metals where this would occur at several meters per second; (ii) at the solidification speed of the droplet experiment, the attachment kinetics anisotropy already dominates the interfacial energy anisotropy, thus making the $\langle 111\rangle$ direction the "easy" growth directions instead of the $\langle 110\rangle$ and totally changing the overall dendritic morphology into some kind of cells.

\subsection{Formation of Cu-rich particles}

It has to be emphasized that the $\mathrm{Cu}$-rich particles form from the liquid phase during the solidification of the droplet and not via a solid state transformation. Several arguments support this statement. 
- First, the particles have a very dendritic morphology as can be seen in Figure 2 or in the detailed EBSD reconstructed map obtained after Ar-ions polishing shown in Fig. 7(a). Again, the twinned grain boundaries between the coarse $\mathrm{Au}$-rich grains are white in this figure and the $\mathrm{Cu}$-rich particles or dendrites are more visible in the zoom of the large brown Au grain shown in (b). The dendritic morphology of the Cu-rich phase is even more apparent in (d) and (e) where two of them have been enlarged. While dendrites can be observed during solid state transformation [32], their formation during cooling of the solid and their large dendrite arm spacing are incompatible with the large cooling rate associated with the FFD experiment.

- Cu-rich dendrites sometimes go across a whole Au-rich grain and clearly appear as patches with the same color/orientation, e.g., in the light brown grain located at the bottom left of the figure. They sometimes even go across Au grain boundaries while keeping the same morphology and orientation, as shown for example in the neighbor pink Au grain. Finally, as revealed by the $\langle 110\rangle$ pole figure of Fig. 7(c), there is no evident crystallographic orientation relationship between the $\mathrm{Cu}$ particles/dendrites and the surrounding Au grain. In this figure, the $\langle 110\rangle$ crystallographic directions of the brown Au grain shown in (b) are identified with small black-brown circles. There are at least 8 or $9 \mathrm{Cu}$ particles/dendrites in this single Au grain, with no clear orientation relationship. Therefore, this Au grain is definitely not the parent phase for the formation of these $\mathrm{Cu}$ particles/dendrites via a solid state transformation.

Interestingly, many of the Cu-rich dendrites exhibit a near-twin or twin relationship, as shown in Fig. 7(d) and (e), respectively. Kurtuldu et al [2] have defined a near-twin relationship as a twin, i.e., rotation of $60 \mathrm{deg}$. around a common $\langle 111\rangle$ direction, followed by a rotation of about $7.5 \mathrm{deg}$. around a common $\langle 110\rangle$ direction. This last rotation is associated with the default aperture angle of $7.5 \mathrm{deg}$, when setting $5 \mathrm{fcc}$ tetrahedra in a decahedral symmetry around a common $\langle 110\rangle$ direction. Although the level of iridium is too low to be detected by EDS, we suspect these atoms to be also responsible of this twinning mechanism via ISRO in the liquid. In Fig. 7(d), twinning seems to start from near the nucleation center of this twinned $\mathrm{Cu}$-rich dendrite, while in (e) the purple and light brown dendrites look like individual entities who have "collided" during growth, an indication that twinning has occurred during growth.

The formation of copper particles is not only related to the presence of iridium in the alloy, but also on the solidification conditions associated with the FFD experiment, i.e. high undercooling and high thermal gradient. No particles were found in the DSC samples nor in alloys processed with FFD without iridium. These $\mathrm{Cu}$-rich particles were also found to be metastable, since the $\mathrm{Cu}$-rich phase can be eliminated by heat treatment.

While there are no known binary, ternary or quaternary phases and metastable phases in the Au-Cu-Ag-Ir system and sub-systems [33], the three binary Au-Ir, Ag-Ir and $\mathrm{Cu}$-Ir systems show a metastable miscibility gap. At high nucleation undercooling, and due to the small solubility of Ir in Au-based alloy (literature values for Ag, $\mathrm{Au}$ and $\mathrm{Cu}$ are reported in Table 2 at the invariant reaction temperature), the liquid phase 


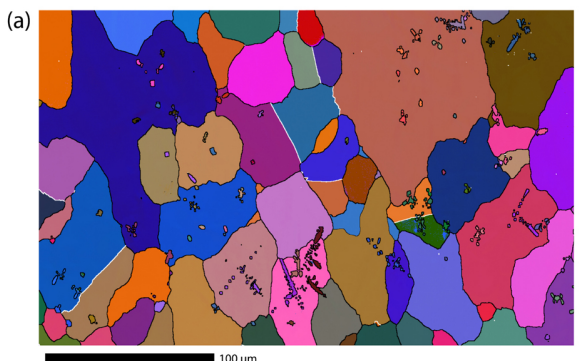

(b)

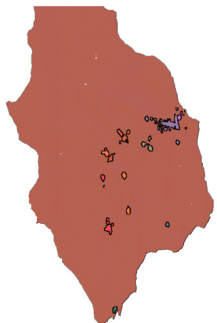

(d)
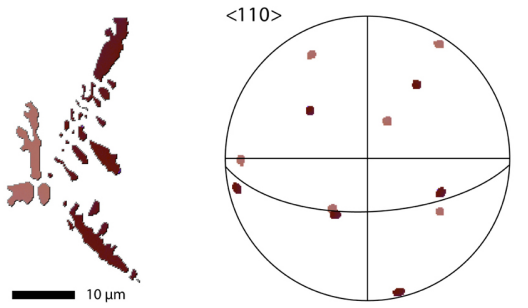

(e)

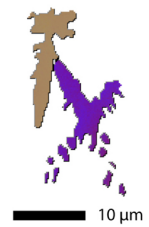

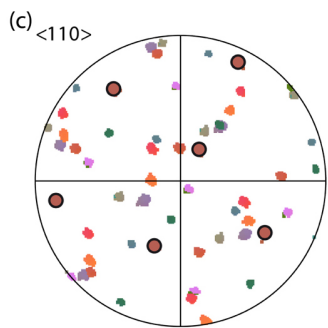

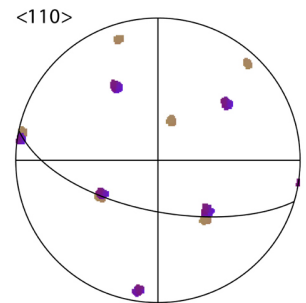

Figure 7: (a) EBSD false color map of the Au-rich grain structure and $\mathrm{Cu}$-rich particles/dendrites obtained on a section of the FFD specimen with Ir after Ar-polishing. The twin grain boundaries between Au-rich grains are shown in white. A zoom of the large brown grain is shown in (b) with its internal $\mathrm{Cu}$-rich particles/dendrites, while the corresponding $\langle 110\rangle$ pole figure is shown in (c) with the same color code. The $\langle 110\rangle$ directions of the Au-rich grain are outlined with small circles. Figures (d) and (e) show two $\mathrm{Cu}$-rich dendrites at other locations of this section with their corresponding $\langle 110\rangle$ pole figure showing that they are near-twinned and twinned, respectively.

can be brought to the miscibility gap region in FFD experiments. Without accurate thermodynamic description of the quaternary system, this issue can not be addressed quantitatively. It can nevertheless be stated that the liquid phase entering the miscibility gap region will undergo a spinodal decomposition process. Using an analogy to Guinier-Preston zones during precipitation, for example in $\mathrm{Al}-\mathrm{Cu}$ alloys, Rappaz and Kurtuldu have also assumed a spinodal decomposition in the Al- $\mathrm{Zn}-\mathrm{Cr}$ system to explain the formation of $\mathrm{Cr}$-centered icosahedra in the liquid in a thermodynamic approach of nucleation [3]. In the present case, the formation of ISRO around iridium atoms would slow down diffusion, as shown by Kurtuldu et al in Al- $\mathrm{Zn}: \mathrm{Cr}$ [14], allowing at high cooling rate the formation of $\mathrm{Cu}$-rich and $\mathrm{Au}$-rich liquid regions, both containing some amount of iridium. Unfortunately, the phase diagram of Au-Ir is totally uncertain (peritectic or eutectic according to [9] or [12], respectively, with very different maximum solubilities), while that for Ag-Ir is eutectic-type and that for $\mathrm{Cu}-\mathrm{Ir}$ is peritectic-type with the largest solubility (see Table 2). From the invariant temperature of the binary X-Ir phase diagrams, it seems natural that iQC-mediated nucleation would occur first in $\mathrm{Cu}$-rich and then in Au-rich liquid regions. $\mathrm{Cu}$-dendrites would then be stopped during their growth by the solidification of the Au-rich regions. 


\section{Conclusions}

Processing Au-20.5wt\%Cu-4.5wt\%Ag alloy with 0.01 Ir addition under rapid solidification conditions has given new insight regarding ISRO and i-QC mediated nucleation. It has been shown that the mechanism previously described by Kurtuldu et al. [1] still applies regardless of the copper and silver composition of the gold alloy. While the number of twins does not evolve significantly with increasing cooling rates, the microstructure is however strongly affected by Ir-induced ISRO of the liquid phase. At high solidification rate, additions of $100 \mathrm{ppm}$ of Ir change the growth morphology from dendrite- to cellular-type as well as the preferred growth direction from standard $\langle 100\rangle$ dendrites to a $\langle 111\rangle$ texture during columnar growth. This correlates the orientation relationship of $\langle 111\rangle_{\mathrm{fcc}} / / \mathrm{i}-3$ axis observed for nucleation, indicating that ISRO also modifies the attachment kinetics.

The most surprising result presented in this paper is the nucleation of primary equiaxed copper particles/dendrites, which are even twinned, when Ir is added to the melt under high cooling rate. It appears that Ir-induced ISRO, by slowing down diffusion, favors a spinodal decomposition of the liquid, with formation of $\mathrm{Cu}$-rich and $\mathrm{Au}$ rich liquid regions containing some iridium. Nucleation and growth in both regions is then influenced as well by ISRO since twinning of $\mathrm{Cu}$-dendrites and multiple-twinning of Au-rich grains are observed.

\section{Acknowledgements}

The authors would like to thank Dr. S. Prades-Rödel and Dr. A. Blatter at PX Group for providing the gold alloys. J.Z. would like to thank E. Etienne for sample preparation. Y. Amal, E. Delon, D. Lacueva Oyarzabala and A. Masson are also acknowledged for their help with heat treatments and associated characterisations.

\section{References}

[1] G. Kurtuldu, A. Sicco, and M. Rappaz: Acta Mater., 2014, vol. 70, pp. 240-48.

[2] G. Kurtuldu, P. Jarry, and M. Rappaz: Acta Mater., 2013, vol. 61(19), pp. 7098108.

[3] M. Rappaz and G. Kurtuldu: JOM, 2015, vol. 67(8), pp. 1812-20.

[4] M. Rappaz and G. Kurtuldu: J.P.E.D., 2016, vol. 37(1, SI), pp. 2-3.

[5] N. Nakayama, K. Tanaka, S. Matsukawa, K. Deguchi, K. Imura, T. Ishimasa, and N.K. Sato: J. Phys. Soc. Japan, 2015, vol. 84, pp. 024721.

[6] S. Matsukawa, K. Tanaka, N. Nakayama, K. Deguchi, K. Imura, H. Takakura, S. Kashimoto, T. Ishimasa, and N.K. Sato: J. Phys. Soc. Japan, 2014, vol. 83, pp. 034705 .

[7] Q. Lin and J.D. Corbett: J. Am. Chem. Soc., 2007, vol. 129, pp. 6789-97. 
[8] I. Karakaya and W.T. Thompson: Binary Alloy Phase Diagrams, II Ed., Ed. T.B. Massalski, 1990, vol. 1, pp. 49-50.

[9] Au-Ir binary phase diagram. http://ipandat.computherm.com/ Diagram/Details/7556, 2017. Accessed: 2017-09-12.

[10] E. Raub and E. Roeschel: Z. Metallkd., 1969, vol. 60, pp. 142-44.

[11] C.J. Essig: Manual for the Use of Dental Students, The S. S. White Dental Manufacturing Co., 1893.

[12] J.P. Nielsen and J.J. Tuccillo: J. Dent. Res., 1966, vol. 45(3), pp. 964-69.

[13] D. Ott and C.J. Raub: Gold Bull., 1981, vol. 14(2), pp. 69-74.

[14] G. Kurtuldu, P. Jarry, and M. Rappaz. Acta Mater., 2016, vol. 115, pp. 423-433.

[15] W. Hornfeck, R. Kobold, M. Kolbe, M. Conrad and D. Herlach: Nat. Commun., 2018, vol. 9, pp. 4054.

[16] S. Wang, D. Daloz, F. Bruneseaux, and G. Lesoult: IOP Conf. Ser. Mater. Sci. Eng., 2012, vol. 27, pp. 012064.

[17] Shuyan Wang: PhD thesis, Université de Lorraine, 2012.

[18] G. Jeanmaire, M. Dehmas, A. Redjaiemia, S. Puech, and G. Fribourg: Mater. Char., 2014, vol. 98, pp. 193-201.

[19] J.O. Andersson, T. Helander, L.H. Hoglund, P.F. Shi, and B. Sundman: Calphad, 2002, vol.26(2), pp. 273-312.

[20] B Sundman, S.G. Fries, and W.A. Oates: Calphad, 1998, vol. 22(3), pp. 335-54.

[21] S. Hassam, J. Ågren, M. Gaune-Escard, and J.P. Bros. Metal. Trans. A, 1990, vol. 21, pp. 1877-84.

[22] A. Kusoffsky: Acta Mater., 2002, vol. 50(20), pp. 5139-45.

[23] A.T. Dinsdale: Calphad, 1991, vol. 15(4), pp. 317-425, 1991.

[24] J.K. Mackenzie: Biometrika, 1958, vol. 45, pp. 229-40.

[25] J.D. Hunt: Mater. Sci. Engng, 1984, vol. 65, pp. 75-83, 1984.

[26] J.A. Dantzig and M. Rappaz: Solidification, 2nd ed., EPFL-Press, Lausanne, Switzerland, 2016.

[27] L. Gránásy, T. Pusztai, J.A. Warren, J.F. Douglas, T. Börzsönyi, and V. Ferreiro: Nature Mater., 2003, vol. 2, pp. 92-96.

[28] S. Henry, T. Minghetti, and M. Rappaz: Acta Mater., 1998, vol. 46, pp.6431-43.

[29] G. Kurtuldu: PhD thesis, EPFL, Lausanne, 2014. 
[30] M. Bedel, G. Reinhart, A.-A. Bogno, C.-A. Gandin, S. Jacomet, E. Boller, H. Nguyen-Thi, and H. Henein: Acta Mater., 2015, vol. 89, pp. 234-246.

[31] J. Ding, M. Asta, and J.J. Hoyt: Frontiers in solidification, TMS Conference, Nashville, USA, 2016. Collection of extended abstracts, Eds W. Kurz et al, wilfried.kurz@epfl.ch.

[32] M. Lima and W. Kurz: Metal. Mater. Trans. A, 2002, vol. 33(8), pp. 2337-45.

[33] Igor L Shabalin: Ultra-High Temperature Materials I, Springer, 2014, pp. 60949. 


\section{List of Figures}

1 (a) Overall and enlarged view and (b) EBSD false colors map showing the microstructure of Au-20.5Cu-4.5Ag:100 wt.ppm Ir solidified in DSC. The view analyzed by EBSD is shown with a white rectangle and the EBSD colors of the grains correspond to their three measured Euler angles. Twin grain boundaries are indicated by white lines. . . .

2 Backscattered electrons image showing the microstructure of $\mathrm{Au}-20.5 \mathrm{Cu}$ - 4.5Ag specimens rapidly solidified without (top) and with (bottom) 100 wt.ppm Ir. The left and right figures correspond to the columnar and equiaxed zones, respectively. For each figure, an enlarged view is shown in the small inserts. . . . . . . . . . . . . . . .

3 False colors EBSD map of $\mathrm{Au}-20.5 \mathrm{Cu}-4.5 \mathrm{Ag}$ rapidly solidified (a) without Ir and (b) with 100 wt.ppm iridium. In (c), the grain sizes deduced from (a) and (b) are plotted as a function of the distance from the copper substrate. The fraction of twinned grain boundaries for the specimen with Ir is also reported in this figure. The vertical dashed lines correspond to the position of the CET with (red) and without (black) Ir additions. The horizontal black line corresponds to the Mackenzie value of twins in a randomly oriented population of grains, with accuracy of $5 \mathrm{deg}$. for their detection. . . . . . . . . . . . . . .

4 (a) False colors EBSD map of 9 nn grains within the equiaxed zone of rapidly solidified $\mathrm{Au}-20.5 \mathrm{Cu}-4.5 \mathrm{Ag}$ with 100 wt.ppm Ir, showing multiple twin or near-twin (MT) relationships compatible with the icosahedron symmetry. Each $\langle 110\rangle$ pole figure shown in (c)-(f) corresponds to a set of 5 (4 in (f)) nn grains having a common $\langle 110\rangle$-axis and MT relationships, i.e., assumed to form on the 5 (4 for (f)) triangular facets of the icosahedron surrounding a 5-fold symmetry axis. Common $\{111\}$ twin or near-twin planes are shown with arcs of circle, while common $\langle 110\rangle$ directions are surrounded with small circles or ellipses. In (b), a comparison is made between the orientation of the common $\langle 110\rangle_{c-f}$ directions of the 4 sets of grains whose pole figures are shown in (c)(f) (blue squares) with the 5-fold symmetry axes of the icosahedron template having the closest orientation (red pentagons). . . . . . . .

5 (a) BSE-SEM image corresponding to Figure 2(b), i.e., Au-20.5Cu4.5 Ag rapidly solidified with 100 wt.ppm Ir, and (b) Surface density of $\mathrm{Cu}$-rich particles/dendrites detected by image analysis. The red dotted line indicates the position of the CET in this specimen. The horizontal dashed black line corresponds to the minimum density of particles measured at $3500 \mu \mathrm{m}$ from the copper substrate. . . . . . . . . . . 
6 Inverse texture pole figures of the columnar zone of the FFD specimens without (a) and with $100 \mathrm{ppm} \operatorname{Ir}(\mathrm{b})$, shown in the unit triangle $\langle 001\rangle$ $\langle 101\rangle-\langle 111\rangle$ of the fcc structure. In these stereographic projections, the grey level indicates the probability of having the thermal gradient direction aligned along a certain crystallographic direction of the Aurich fcc grains. The texture density is scaled between 0 and 10 for both specimens. . . . . . . . . . . . . . . . .

7 (a) EBSD false color map of the Au-rich grain structure and Cu-rich particles/dendrites obtained on a section of the FFD specimen with Ir after Ar-polishing. The twin grain boundaries between Au-rich grains are shown in white. A zoom of the large brown grain is shown in (b) with its internal $\mathrm{Cu}$-rich particles/dendrites, while the corresponding $\langle 110\rangle$ pole figure is shown in (c) with the same color code. The $\langle 110\rangle$ directions of the Au-rich grain are outlined with small circles. Figures (d) and (e) show two Cu-rich dendrites at other locations of this section with their corresponding $\langle 110\rangle$ pole figure showing that they are neartwinned and twinned, respectively. . . . . . . . . . . . 


\begin{tabular}{lccc}
\hline \multirow{2}{*}{ Alloy } & $\begin{array}{c}\dot{T} \\
(\mathrm{~K} / \mathrm{s})\end{array}$ & $\begin{array}{c}\text { Twin frequency } \\
(\%)\end{array}$ & $\begin{array}{c}\text { Grain size } \\
(\mu \mathrm{m})\end{array}$ \\
\hline \multirow{2}{*}{ Au-Cu-Ag } & -0.25 & $\star$ & $2000^{\star}$ \\
& -100 & $0.13 \pm 0.17$ & $99 \pm 68$ \\
\hline \multirow{2}{*}{ Au-Cu-Ag:Ir } & -0.25 & $7.58 \pm 0.17$ & $125 \pm 38$ \\
& -100 & $7.50 \pm 0.09$ & $27 \pm 15$
\end{tabular}

Table 1: Average twin boundary fraction and grain size in the equiaxed zone of the samples with/without Ir, solidified at low/high cooling rate. ( ${ }^{\star}$ only two grains were found in this sample). The error associated with the twin frequency is calculated with a $99 \%$ confidence interval, the error associated with the grain size corresponds to the standard deviation given by the EBSD measurements.

\begin{tabular}{lccccc}
\hline System & $\mathrm{C}_{\text {inv }}^{\text {liq }}$ & $\mathrm{C}_{\text {inv }}^{\text {fcc }}$ & $\mathrm{T}_{\text {inv }}\left[{ }^{\circ} \mathrm{C}\right]$ & Inv. type & Ref. \\
\hline $\mathrm{Ag}-\mathrm{Ir}$ & $<0.3$ & $<0.3$ & 961 & eut. & {$[8]$} \\
$\mathrm{Au}-\mathrm{Ir}$ & 0.1 & 0.46 & 1069 & per. & {$[9]$} \\
$\mathrm{Au}-\mathrm{Ir}$ & 0.005 & 0.002 & 1056 & eut. & {$[12]$} \\
$\mathrm{Cu}-\mathrm{Ir}$ & 4.0 & 6.0 & 1138 & per. & {$[10]$} \\
\hline
\end{tabular}

Table 2: Solubility values of Ir (at.\%) in liquid and fcc solutions for the binary systems of interest at the invariant reaction temperature (eut.: eutectic, per.: peritectic). For comparison, the liquidus of $\mathrm{Au}-20.5 \mathrm{Cu}-4.5 \mathrm{Ag}$ is $900^{\circ} \mathrm{C}$. 